\title{
Genetics of Resistance to Transgenic Bacillus thuringiensis Poplars in Chrysomela tremulae (Coleoptera: Chrysomelidae)
}

\author{
SYLVIE AUGUSTIN,${ }^{1}$ CLAUDINE COURTIN,${ }^{1}$ AGNES REJASSE, ${ }^{2}$ PHILIPPE LORME, ${ }^{1}$ \\ ANNE GENISSEL, ${ }^{1,3}$ AND DENIS BOURGUET ${ }^{2,4}$
}

\begin{abstract}
J. Econ. Entomol. 97(3): 1058-1064 (2004)
ABSTRACT The area under genetically engineered plants producing Bacillus thuringiensis (Bt) toxins is steadily increasing. This increase has magnified the risk of alleles conferring resistance to these toxins being selected in natural populations of target insect pests. The speed at which this selection is likely to occur depends on the genetic characteristics of Bt resistance. We selected a strain of the beetle Chrysomela tremulae Fabricius on a transgenic Bt poplar clone Populus tremula L. x Populus tremuloides Michx producing high levels of B. thuringiensis Cry3Aa toxin. This strain was derived from an isofemale line that generated some $\mathrm{F}_{2}$ offspring that actively fed on this Bt poplar clone. The resistance ratio of the strain was $>6,400$. Susceptibility had decreased to such an extent that the mortality of beetles of the strain fed Bt poplar leaves was similar to that of beetles fed nontransgenic poplar leaves. Genetic crosses between susceptible, resistant, and F1 hybrids showed that resistance to the Cry3Aa toxin was almost completely recessive $\left(D_{L C}=0.07\right)$ and conferred by a single autosomal gene. The concentration of Cry3Aa produced in the transgenic Bt poplar used in this study was 6.34 times higher than the $\mathrm{LC}_{99}$ of the $\mathrm{F} 1$ hybrids, accounting for the complete recessivity $\left(D_{M L}=0\right)$ of survival on Bt poplar leaves. Overall, the genetic characteristics of the resistance of $C$. tremulae to the Cry3Aa toxin are consistent with the assumptions underlying the high-dose refuge strategy, which aims to decrease the selection of Bt resistance alleles in natural target pest populations.
\end{abstract}

KEY WORDS Chrysomela tremulae, Bt resistance, dominance level, Bacillus thuringiensis, transgenic poplar

ReSISTANCE to Bacillus thuringiensis (Bt) toxins has been extensively studied, particularly since the development of genetically modified plants containing genes encoding these toxins. These plants, referred as to Bt plants, provide a safe and effective method for controlling some of their pests. Although Bt maize, $\mathrm{Bt}$ cotton, and Bt potatoes account for $>90 \%$ of the planted Bt crops, many other Bt plant species are available. The area under Bt plants $(\approx 10$ million ha in 2002; James 2003) is expanding every year, increasing the risk of rapid selection for resistance alleles in targeted insect pest species (Gould 1998, Wolfenbarger and Phifer 2000, Tabashnik et al. 2003). To minimize this risk, the U.S. Environmental Protection Agency, in collaboration with various stakeholders, has encouraged growers to implement an effective

\footnotetext{
${ }^{1}$ Institut National de la Recherche Agronomique, Centre de Recherches d'Orléans, Unité de Zoologie Forestière, BP 20619 Ardon, 45 166 Olivet, France.

${ }^{2}$ Institut National de la Recherche Agronomique, Unité Génétique Microbienne et Environnement, La Minière, 78285 Guyancourt Cedex, France.

${ }^{3}$ Current address: Ecole Pratique des Hautes Etudes, Laboratoire d'Ecologie, Université Pierre et Marie Curie, 75252 Paris cedex 05, France.

${ }^{4}$ Centre de Biologie et de Gestion des Populations (CBGP), Institut National de la Recherche Agronomique, Campus International de Baillarguet, CS 30 016, 34988 Montferrier/Lez, France.
}

insecticide resistance management (IRM) strategy known as the high-dose plus refuge (HDR) strategy (EPA 2001).

The HDR strategy was originally proposed by Georghiou and Taylor (1977) for managing the evolution of resistance to conventional insecticides. However, this strategy was not used in previous IRM plans because the doses and concentrations of insecticides cannot be controlled over time (insecticides are quickly diluted by rain), and space (insecticides are not evenly distributed over the plants and do not penetrate plant tissues). As pointed out by Alstad and Andow (1995), the possibility of engineering genetically modified crops producing high doses of Bt toxin offers a unique opportunity to use the HDR strategy to delay the occurrence of widespread resistance.

The production of a high dose of toxin by the plant tissues is one of the most important components of the HDR strategy. Indeed, the efficacy of this strategy depends on the concentration of $\mathrm{Bt}$ toxin produced by the Bt plant being sufficiently high to kill heterozygous individuals (Andow and Alstad 1998). In other words, resistance to Bt toxins must be recessive so that only resistant homozygotes can complete their life cycle on Bt plants. This assumption has not often been evaluated because such evaluation requires the selection of alleles in the pest species that confer sufficient 
resistance for survival on the Bt plants. Although several Bt-resistant strains have been selected in various crop pest species (reviews by Ferré and Van Rie 2002 , Tabashnik et al. 2003), few can actively feed on Bt crops. For example, none of the laboratory experiments performed on the European corn borer, Ostrinia nubilalis (Hübner) (Lepidoptera: Crambidae), has resulted in the successful selection of alleles conferring resistance to $\mathrm{Bt}$ corn producing the $\mathrm{Bt} \mathrm{Cryl} \mathrm{Ab}$ toxin (Huang et al. 2002, Bourguet et al. 2003).

In a field population of the leaf beetle Chrysomela tremulae Fabricius (Coleoptera: Chrysomelidae), we recently detected individuals that were able to complete their life cycle on transgenic Bt poplar Populus tremula L. x Populus tremuloides Michx producing the Cry3Aa toxin (Génissel et al. 2003a). These individuals were detected in three isofemale lines $(>270$ lines were tested). From these results, we estimated the mean resistance allele frequency for the period 1999 2001 to be 0.0037 (Génissel et al. 2003a). We report here an analysis of a strain of $C$. tremulae derived from one of these three resistant isofemale lines. Using multiple concentrations of purified Cry3Aa toxin and screening for survival on Bt poplars, we 1) estimated the ratio of resistance to Cry3Aa, 2) calculated dominance levels, and 3) determined whether the resistance to Cry3Aa was conferred by a single autosomal gene.

\section{Materials and Methods}

Insects. Two strains of C. tremulae were obtained from adults collected in a poplar plantation at Vatan (Centre region of France). The susceptible (S\#125) strain originated from the offspring of an isofemale line devoid of alleles conferring resistance to the Cry3Aa toxin (Génissel et al. 2003a). This strain has been maintained in the laboratory without exposure to Bt toxin for 15 generations. The resistant strain $(\mathrm{R} \# 60)$ was established from one of the resistant isofemale line selected on transgenic Bt poplar by Génissel et al. (2003a), by using the $\mathrm{F}_{2}$ screen method described by Andow and Alstad (1998). Selected males from this line were crossed with virgin females from the susceptible strain S\#125. F1 progeny were pooled and mass crossed. The F2 neonates were selected on Bt27 poplar leaves, giving rise to the resistant strain $\mathrm{R} \# 60$. Strains were reared in laboratory conditions at $20^{\circ} \mathrm{C}$, with a photoperiod of 16:10 (L:D) h. The susceptible strain was fed fresh leaves from a nontransgenic poplar hybrid clone (Populus tremula $\times$ Populus tremuloides, INRA \#353-38). The neonate larvae of the resistant strain were fed for $4 \mathrm{~d}$ on fresh transgenic poplar leaves and were then transferred to nontransgenic foliage.

Bt Poplar and Bt Toxin Cry3Aa. Bioassays were performed with either the transgenic Bt poplar line $B t 27$ (referred as to Bt27 poplar) or purified Cry3Aa crystal preparations. Bt27 poplar was obtained by transformation of the poplar hybrid clone INRA \#35338, by using Agrobacterium tumefasciens. This line expressed a synthetic cry $3 \mathrm{Aa}$ gene from $B$. thuringiensis (Génissel et al. 2003b). Both transgenic and nontransgenic poplars used were grown in greenhouses. Toxin levels in the leaves of $B t 27$ poplar were high enough to kill all susceptible C. tremulae neonates within $48 \mathrm{~h}$ of feeding (Génissel et al. 2003a, b).

Purified Cry3Aa crystals were prepared from an asporogenic Bt strain $407^{-}$Spo 0A (pHT 305P) harboring the cry3Aa gene from the LM 79 strain (Chaufaux et al. 1993). The Bt strain was grown overnight at $30^{\circ} \mathrm{C}$, with shaking at $150 \mathrm{rpm}$, from a single colony, in Luria broth supplemented with selective antibiotics ( 5 $\mu \mathrm{g} / \mathrm{ml}$ erythromycin and $100 \mu \mathrm{g} / \mathrm{ml}$ kanamycin). When the optical density at $600 \mathrm{~nm}$ reached 3 , we removed $10 \mu \mathrm{l}$ of this preculture to another flask, to which we added $100 \mathrm{ml}$ of casein tryptone hydrolysate and $3 \%$ of glucose. We cultured the Bt strain at $30^{\circ} \mathrm{C}$ with shaking at $150 \mathrm{rpm}$ for $48 \mathrm{~h}$, until crystal formation was observed. Cultures were then washed twice with $0.15 \mathrm{M} \mathrm{NaCl}$ and twice with distilled water, and sonicated for $10 \mathrm{~min}$ on ice to release the crystals. The crystals were freeze-dried for $24 \mathrm{~h}$ and resuspended in water. Protein concentration was determined with the Bio-Rad protein assay (Bio-Rad $\mathrm{GmbH}$, München, Germany) and the size of toxin was determined by SDS-polyacrylamide gel electrophoresis (10\%).

Bioassays with Bt27 Poplars. Neonates emerging from egg masses were fed on leaf discs $(2 \mathrm{~cm}$ in diameter) cut from fresh mature leaves of $B t 27$ poplars. These leaf discs were placed on moist filter paper in petri dishes to prevent them from drying out. Susceptible C. tremulae neonates cannot survive on $\mathrm{Bt}$ poplar leaves for $>2 \mathrm{~d}$ (Génissel et al. 2003b). Hence, after $4 \mathrm{~d}$, the surviving larvae, which had actively fed on Bt poplar, were considered to be resistant.

Bioassays with Purified Cry3Aa Preparations. Leaf discs $(2 \mathrm{~cm}$ in diameter) were cut from fresh nontransgenic poplar hybrid clones INRA \#353-38. These discs were treated with various serial dilutions of the purified Cry3Aa crystal preparation. These preparations were diluted in $0.2 \%$ Triton $\mathrm{X}-100$ and leaf discs treated with $0.2 \%$ Triton $\mathrm{X}-100$ only were used as a control. We deposited $30 \mu \mathrm{l}$ of solution on each side of the leaf and spread the solution over the entire area of the leaf disc. The solution was allowed to dry on the leaf surface in the air, and the leaf discs were then placed on moist filter paper in petri dishes to ensure that they did not dry out. At least seven replicates were carried out for each dilution and for the control. The only exception was for $\mathrm{BC} 4$ for which five replicates were carried out for toxin concentrations of 42.02 and $2,100 \mathrm{ng} / \mathrm{cm}^{2}$. Each replicate consisted of seven neonates placed on a single disc and new discs were added if the insects ran out of leaf material. Mortality was recorded after $4 \mathrm{~d}$ of exposure.

Cry3Aa Concentration in Bt27 Poplar Leaves. Two fully expanded leaves were collected from four Bt27 poplars and from two nontransgenic poplar hybrid clones (INRA \#353-38). Each leaf was weighed and scanned independently before analysis. We used $4 \mathrm{ml}$ of CAPS buffer, $\mathrm{pH} 10.0$, supplemented with the $\mathrm{L}^{-}(+)$ sodium salt of ascorbic acid at a concentration of 200 $\mathrm{mM} / \mathrm{g}$ of leaf for protein extraction. Leaves were ground with a Polytron PCU (Fisher Bioblock Scientific, Illkirch, France) for 5 min, the resulting homog- 
enate was filtered, and the filtrates were centrifuged at $16,440 \times \mathrm{g}$ twice, $10 \mathrm{~min}$ each, at $4^{\circ} \mathrm{C}$. The supernatant was stored at $-80^{\circ} \mathrm{C}$.

Cry 3Aa protein levels were monitored by enzymelinked immunosorbent assays. We used the indirect peroxidase detection method, assessing a range of concentrations of a standard (purified toxin 7.5 and 120 ng) for comparison. The sample and the purified Cry3Aa toxin were prepared in coating buffer $(50 \mathrm{mM}$ $\mathrm{NaHCO}_{3}, \mathrm{pH}$ 9.6) and used to coat microtiter plates. The plates were then incubated with the primary rabbit anti-Cry3Aa antibody, at a dilution of $1 / 5,000$. The plates were washed with buffer Tris-Tween (20 $\mathrm{mM}, \mathrm{pH} 7.4+$ bovine serum albumin at $1 \mathrm{~g} /$ liter) and the secondary peroxidase-conjugated goat antibody was added, at a dilution of $1 / 5,000$. Plates were incubated with ABTS for $1 \mathrm{~h}$ at room temperature, and absorbance at $405 \mathrm{~nm}$ was read on a Labsystems Uniskan II machine.

Genetics of Cry3Aa Resistance. We investigated the genetics of C. tremulae resistance to Cry3Aa by mass mating males with virgin females of the appropriate strain or F1 offspring. Virgin females were obtained by isolating individual pupae and sexing adults as soon as they emerged. Hybrid F1 offspring were generated by crossing resistant males with susceptible females (i.e., o $\mathrm{S} \# 125 \times$ o $\mathrm{R} \# 60$ ). We used these $\mathrm{Fl}$ offspring for the following backcrosses: 1) two backcrosses between F1 and susceptible individuals: BC1 ( + F1 $\times$ o $\mathrm{S} \# 125$ ) and $\mathrm{BC} 2$ ( $9 \mathrm{~S} \# 125 \times \widehat{\mathrm{O}} \mathrm{F} 1$ ); and 2) two backcrosses between $\mathrm{F} 1$ and resistant individuals: BC3

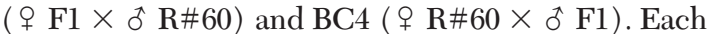
cross or backcross involved the mass mating of 20 females and at least three males.

Data Analysis. For bioassays with various concentrations of purified Cry3Aa toxin, we used probit analysis to estimate $\mathrm{LC}_{50}$ values and their $95 \%$ confidence interval (CI), the slopes of concentration-mortality curves, and their standard errors. Resistance ratios were calculated by dividing the $\mathrm{LC}_{50}$ for a strain or a cross by the $\mathrm{LC}_{50}$ for the susceptible strain $\# 125 \mathrm{~S}$. Regression lines were considered identical if the hypothesis of parallelism was not rejected at the 0.05 probability level (in a chi square test; Finney 1971) and if the $95 \%$ CI of the resistance ratio included 1.0 (Robertson and Priesler 1992). Analyses were performed with the Probit software developed by Raymond et al. (1993). Dominance levels were calculated from either $L_{50}$ values $\left(D_{L C}\right)$ or from single-dose mortality levels $\left(D_{M L}\right)$ as described by Bourguet et al. (2000). $D_{L C}$ was calculated as $=\left(\operatorname{logLC} C_{F 1}-\right.$ lo$\left.\operatorname{gLC}_{\mathrm{S} \# 125}\right) /\left(\operatorname{logLC} \mathrm{R} \# 60_{1}-\operatorname{logLC} \mathrm{S}_{\mathrm{S} 125}\right)$ and $D_{M L}=$ $\left(\mathrm{ML}_{\mathrm{F} 1}-\mathrm{ML}_{\mathrm{S} \# 125}\right) /\left(\mathrm{ML}_{\mathrm{R} \# 60}-\mathrm{ML}_{\mathrm{S} \# 125}\right)$, where $\mathrm{ML}_{\mathrm{S} \# 125}, \mathrm{ML}_{\mathrm{F} 1}$, and $\mathrm{ML}_{\mathrm{R} \# 60}$ are the mortality levels of susceptible, F1 hybrid, and resistant neonates on Bt27 poplar leaves, respectively. Mortality levels were corrected for control mortality, using Abbot's formula (Abbot 1981). The range of $D_{L C}$ and $D_{M L}$ is 0 (complete recessivity) to 1.0 (complete dominance). Finally, Fisher exact and chi square tests were used to determine whether the mortality levels of backcross progeny on Bt27 poplar were significantly different from those expected assuming monogenic inheritance of the resistance.

\section{Results}

Resistance Ratio of the Bt-Resistant Strain. The concentration-mortality curve obtained with the purified Cry3Aa toxin was linear $\left(\chi^{2}=1.357, \mathrm{df}=4, P=0.852\right)$ for the susceptible $\mathrm{S} \# 125$ strain (Fig. 1), indicating homogeneity for tolerance in this strain. The $\mathrm{LC}_{50}$ was $31.1 \mathrm{ng} / \mathrm{cm}^{2}$ (95\% CI 27.0-34.7) (Table 1). As expected from previous results (Génissel et al. 2003b), all neonates $(n=100)$ on Bt27 poplar leaves died within $48 \mathrm{~h}$ (Table 2$)$. In contrast, only $16.67 \%(n=$ 90 ) of resistant $R \# 60$ neonates died after feeding for $4 \mathrm{~d}$ on these transgenic poplar leaves. This mortality rate did not differ significantly $\left(\chi^{2}=0.054, \mathrm{df}=1, P=\right.$ $0.816)$ from that $(15.45 \%, n=110)$ on nontransgenic poplar leaves. The corrected mortality rate was $1.1 \%$.

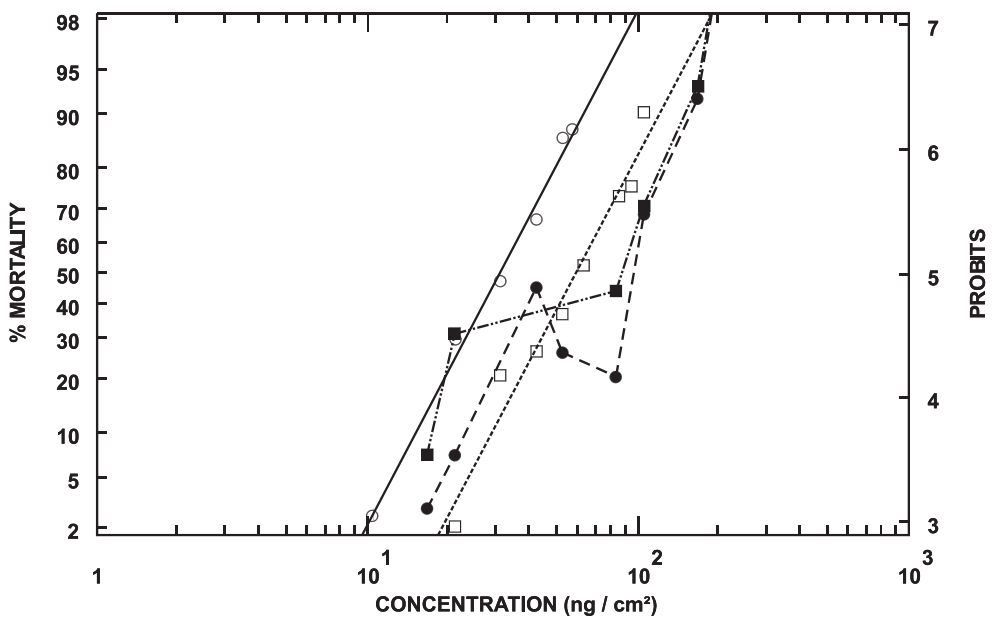

Fig. 1. Concentration-mortality curves obtained in bioassays of Cry3Aa toxin with larvae of the susceptible S\#125 strain $(\bigcirc)$, the F1 hybrid $(\square)$, and backcrosses [BC1 $(\mathbf{O})$ and BC2 ( $\square)]$ of C. tremulae. 
Table 1. Responses of susceptible (S\#125), resistant (R\#60), and hybrid F1 progenies of C. tremulae to the Cry3Aa Bt toxin

\begin{tabular}{lccc}
\hline \multicolumn{1}{c}{ Strain or cross } & No. of larvae & Slope \pm SE & LC $_{50}(95 \% \mathrm{CI}) \mathrm{in} \mathrm{ng} / \mathrm{cm}^{2}$ \\
\hline S\#125 & 364 & $3.9 \pm 0.5$ & $31: 1(27.0-34.7)$ \\
R\#60 & 266 & NA & $>199,370(\text { NA })^{b}$ \\
F1 (q S\#125 $\times$ o R\#60) & 581 & $4.1 \pm 0.4$ & $58.8(54.2-63.2)$ \\
\hline
\end{tabular}

NA, not available.

${ }^{a}$ Resistance ratio $\left(\mathrm{LC}_{50}\right.$ for the strain or cross divided by $\mathrm{LC}_{50}$ for the $\mathrm{S} \# 125$ strain)

${ }^{b}$ The highest concentration $\left(199 \mu \mathrm{g} / \mathrm{cm}^{2}\right)$ of Cry3Aa toxin killed $18 \%$ of the $\mathrm{R} \# 60$ neonates.

We were unable to estimate the $\mathrm{LC}_{50}$ of the resistant strain (Table 1) because large numbers of $\mathrm{R} \# 60$ individuals survived the highest possible concentration of Cry3Aa toxin we could make. The highest dose tested caused only $18 \%$ mortality, indicating that the $\mathrm{LC}_{50}$ of the $\mathrm{R} \# 60$ strain was at least 6,410 times greater than the $\mathrm{LC}_{50}$ of the susceptible strain \#125 (Table 1).

Dominance Levels. The $\mathrm{LC}_{50}$ of the F1 hybrid ( $q$ $\mathrm{S} \# 125 \times$ ○ $\mathrm{R} \# 60)$ progeny was $58.8 \mathrm{ng} / \mathrm{cm}^{2}(95 \% \mathrm{CI}$ $54.2-63.2)$. The F1 concentration-mortality curve was linear $\left(\chi^{2}=5.799, \mathrm{df}=6, P=0.446\right)$, but not parallel $\left(\chi^{2}=66.074, \mathrm{df}=10, P<10^{-4}\right)$ to the $\mathrm{S} \# 125$ mortality line. The resistance ratio was 1.89 and the $95 \%$ CI (1.58-2.27) did not include 1.0. Given that the resistance ratio of the resistant strain is $>6,410$ (Table 1 ), the dominance of $\mathrm{Bt}$ resistance $\left(D_{L C}\right)$ was $<0.07$. Hence, the Cry 3Aa resistance gene selected in the $\mathrm{R} \# 60$ strain is almost completely recessive.

All F1 hybrid neonates $(n=150)$ feeding on Bt27 poplar leaves died within $2 \mathrm{~d}$ (mortality on nontransgenic poplar leaves was $6.12 \%[n=147])$. Based on the mortality levels of susceptible, resistant and F1 neonates on Bt27 poplar leaves, we calculated the dominance level $D_{M L}=0$. Hence, resistance to Bt27 poplars is completely recessive.

Concentration of Cry3Aa toxin in Transgenic Leaves. The leaves of Bt27 poplar contained 1,362 \pm 148 (mean $\pm \mathrm{SE}$ ) $\mathrm{ng} / \mathrm{cm}^{2}$ Cry $3 \mathrm{Aa}$ protein, corresponding to $1.25-2.75 \%$ of the total soluble protein. Cry3Aa was not detected in nontransgenic leaves (details not shown). Susceptible and hybrid neonates were unable to survive Cry3Aa concentrations $>300$ $\mathrm{ng} / \mathrm{cm}^{2}$ (Fig. 1). Thus, the failure of susceptible and hybrid neonates to survive on Bt27 poplar leaves is perfectly accounted for by the high concentration of
Cry3Aa toxin within transgenic leaves. These results are also consistent with the survival of resistant larvae on Bt27 poplar.

Inheritance of Bt Resistance. If susceptibility to Cry3Aa is controlled by a single autosomal gene, then susceptible $\mathrm{S} \# 125$, resistant $\mathrm{R} \# 60$, and F1 hybrid individuals would have the following genotypes: SS, RR, and RS, respectively. Under this assumption, the BC1 and BC2 progeny should contain $50 \%$ SS and $50 \%$ RS, whereas the BC3 and BC4 progeny should contain $50 \%$ RS and $50 \%$ RR. We tested this hypothesis by evaluating the susceptibility of backcross progeny to various concentrations of Cry $3 \mathrm{Aa}$ and to Bt27 poplar leaf discs.

The range of Cry $3 \mathrm{Aa}$ concentrations killing between 0 and $100 \%$ of $\mathrm{BC} 1$ and $\mathrm{BC} 2$ neonates was between the $\mathrm{LC}_{2}$ of the susceptible strain and the $\mathrm{LC}_{99}$ of the F1 hybrid progeny (Fig. 1). Neither the BC1 mortality curve nor that for BC2 was linear $\left(P<10^{-4}\right)$. As expected under an assumption of a monofactorial inheritance of Cry3Aa resistance, both mortality curves reached a plateau between the mortality curves for S\#125 and F1 hybrids (Fig. 1). Surprisingly, for $\mathrm{BCl}$, the toxin concentration of $42.02 \mathrm{ng} / \mathrm{cm}^{2}$ induced a higher mortality rate than toxin concentrations of 52.52 and $84.03 \mathrm{ng} / \mathrm{cm}^{2}$. This difference was due to three replicates in which only one, zero, and one larva survived. Removing these replicates, mortality rates were $34.69 \%(n=49), 33.93 \%(n=56)$ and $28.57 \%$ $(n=70)$ at toxin concentrations of $42.01,52.52$, and $84.03 \mathrm{ng} / \mathrm{cm}^{2}$, respectively. These mortality rates were not significantly different from each other (Fisher exact test on contingency table, $P=0.742$ ).

The lowest Cry3Aa concentration killing $0-50 \%$ of the BC3 and BC4 progeny was similar to that that killed the F1 hybrid larvae (Fig. 2), consistent with the

Table 2. Mortality levels on $B t 27$ poplar leaves and test for deviation from expected mortality under the assumption that resistance to Cry3Aa is recessive and monogenic

\begin{tabular}{|c|c|c|c|c|c|c|}
\hline \multirow{2}{*}{ Strain or cross } & \multirow{2}{*}{$\begin{array}{l}\text { No. of } \\
\text { larvae }\end{array}$} & \multicolumn{2}{|c|}{ Mortality (\%) } & \multicolumn{3}{|c|}{$\begin{array}{l}\text { Tests for deviation between observed } \\
\text { and expected mortality }\end{array}$} \\
\hline & & Observed $^{a}$ & Expected $^{b}$ & $\chi^{2}$ & df & $P$ value \\
\hline $\mathrm{S} \# 125$ & 100 & 100 & 100 & & & \\
\hline $\mathrm{R} \# 60$ & 90 & 1.1 & 0 & & & \\
\hline $\mathrm{F} 1($ 오 $\mathrm{S} \# 125 \times \delta \mathrm{R} \# 60)$ & 150 & 100 & 100 & & & \\
\hline 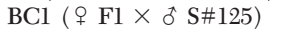 & 240 & 100 & 100 & & & \\
\hline $\mathrm{BC} 2($ 오 $\mathrm{S} \# 125 \times$ × $\mathrm{F} 1$ ) & 154 & 100 & 100 & & & \\
\hline BC3 $(ㅇ ㅏ 1 \times \delta ~ R \# 60)$ & 186 & 47.3 & 50 & 0.538 & 1 & 0.463 \\
\hline $\mathrm{BC} 4($ ( ㅇ $\mathrm{R} \# 60 \times$ ơ $\mathrm{F} 1$ ) & 209 & 61.7 & 50 & 11.488 & 1 & $<10^{-3}$ \\
\hline
\end{tabular}

\footnotetext{
${ }^{a}$ Mortality rates were corrected for mortality on nontransgenic hybrid poplar leaves using Abbot's formula (Abbot 1981). Mortality rate on nontransgenic hybrid poplar leaves was estimated for at least 110 individuals and was always $<9.66 \%$.

${ }^{b}$ Expected mortality rate under the assumption that resistance is monogenic and recessive (i.e., resistance conferred by a single gene).
} 


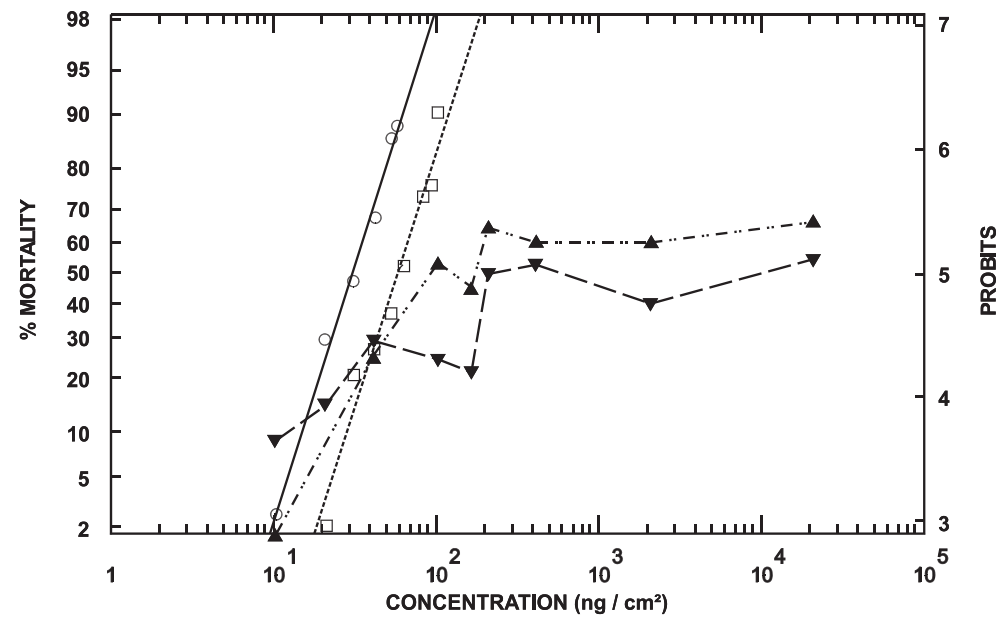

Fig. 2. Concentration-mortality curves obtained in bioassays of Cry3Aa toxin with larvae of the susceptible S\#125 strain $(\bigcirc)$, the F1 hybrid $(\square)$, and backcrosses [BC3 $(\boldsymbol{\nabla})$ and BC4 $(\mathbf{\Delta})]$ of C. tremulae.

presence of RS larvae. For both backcrosses, mortality increased and then reached a plateau at $\approx 50 \%$ mortality (Fig. 2). Mortality levels were similar for concentrations of $210,420,2,100$, and $21,000 \mathrm{ng}$ toxin $/ \mathrm{cm}^{2}$ (Fisher exact tests: $P>0.110$ for BC3 and $P>0.559$ for BC4) and were not significantly different from $50 \%$ for BC3 ( $\chi^{2}$ tests, $P>0.05$ for each concentration). For BC4, mortality rates at concentrations of 210,420 , and $21,000 \mathrm{ng} / \mathrm{cm}^{2}$ were slightly, but significantly higher than $50 \%$ ( $\chi^{2}$ tests, $P<0.05$ for all three concentrations), whereas that at a concentration of 2,100 ng/ $\mathrm{cm}^{2}$ was not $\left(\chi^{2}=2.332, \mathrm{df}=1, P=0.127\right)$. Nevertheless, at toxin concentrations of $210,420,2,100$, and $21,000 \mathrm{ng} / \mathrm{cm}^{2}$ mortality rates of BC3 and BC4 were not significantly different from each other $\left(\chi^{2}\right.$ tests, $P>0.05$ for each concentration). Even the highest concentration of Cry3Aa did not kill $>50 \%$ of the BC3 and BC4 larvae (Fig. 2). Hence, the BC3 and BC4 mortality curves are consistent with what would be expected if the BC3 and BC4 progeny each consisted of a 50:50 mixture of RS and RR larvae.

The mortality rate on nontransgenic poplar leaves was $3.45 \%(n=232)$ for $\mathrm{BCl}$ and $4.51 \%(n=155)$ for BC2. As expected under the assumption of monofactorial inheritance of Cry3Aa resistance, the progeny of these two backcrosses were unable to survive on Bt27 poplar leaves (Table 2). For BC3, the mortality rate on Bt27 poplar leaves (corrected for the mortality rate on nontransgenic leaves: $5.95 \%[n=168])$ was $47.3 \%$ (95\% CI 39.7-54.7), which is consistent $\left(\chi^{2}=0.538\right.$, $\mathrm{df}=1, P=0.463)$ with the expected proportion of $50 \%$ (Table 2). Finally, for BC4, after correction for the mortality rate on nontransgenic poplar leaves $(9.66 \%$, $n=176)$, the residual mortality rate was $61.7 \%(95 \%$ CI 54.8-68.5), which is significantly $\left(\chi^{2}=11.488, \mathrm{df}=\right.$ $\left.1, P<10^{-3}\right)$ higher than the expected proportion of $50 \%$ (Table 2). Mortality rates of BC3 and BC4 were significantly different from each other (Fisher exact test, $P=0.0042$ ), indicating an effect of the sex used for the backcrosses. This effect may be due to either a maternal effect changing the susceptibility of RR individuals or a difference in the relative proportion of RR individuals within the progeny tested on Bt poplar.

A maternal effect causing $\mathrm{RR}$ individuals to be partly susceptible to the concentration of Cry3Aa produced by Bt poplar leaves is less likely than the other proposition proposed. The reason is that the difference in mortality rate between $\mathrm{BC} 4$ and $\mathrm{BC} 3$ remained constant at all concentrations of Cry3Aa even at the highest concentration (Fig. 2). A maternal effect decreasing by $\approx 10 \%$ the survival rate of $\mathrm{RR}$ individuals at all concentrations of toxin is difficult to explain. A parsimonious explanation is that, compared with BC3, the progeny of BC4 had a lower proportion of RR individuals than expected $(\approx 40 \%$ rather than $50 \%$ ). One possibility is that due to a nondominant fitness costs expressed during the early stage of the development some RR individuals of the BC4 progeny died before being tested on Bt poplar or in Cry3Aa bioassays. A second possibility is that a distortion of segregation occurred in BC4 leading to a lower proportion of RS than expected.

In conclusion, mortality curves and survival on Bt27 poplar leaf discs suggest that the Cry3Aa resistance selected in the $R \# 60$ strain is conferred by a single autosomal gene, with some evidence that the relative proportion of RR individuals in some backcross progeny can be lowered due to nondominant fitness costs expressed during the early stage of the development and/or distortion of segregation.

\section{Discussion}

Our results indicate that the susceptibility of the $\mathrm{R} \# 60$ strain to the Cry3Aa toxin is lower than that of the S\#125 strain by a factor of $>6,400$. L. S. Bauer (unpublished data) previously carried out selection with Cry3A in a population of the closely related species Chrysomela scripta F. After 41 generations of selection, she obtained an $\mathrm{RR}>5,000$. Based on the 
sample size ( $n=110$; L. S. Bauer, personal communication) of the natural population from which her resistant strain was selected and assuming that at least one of the founders was heterozygous (RS), we can estimate the frequency of the Cry $3 \mathrm{~A}$ resistance allele in Bauer's population from the United States to be 0.0045 . This frequency is similar to that found in a natural population of $C$. tremulae by Génissel et al. (2003a). Indeed, the Cry3Aa resistance allele displayed by the $R \# 60$ strain was found to segregate at a frequency of 0.0037 in a poplar plantation in the Centre region of France. Hence, it would be interesting to establish whether the molecular basis of resistance to Cry3Aa selected in these two species of Chrysomelidae is similar. The RR in C. tremulae and C. scripta are among the highest obtained to date by deliberate selection (reviews in Ferré and Van Rie 2002, Tabashnik et al. 2003). We show here that this RR is high enough to render C. tremulae resistant to a transgenic poplar expressing the cry3Aa gene that was engineered by Génissel et al. (2003b). Indeed, larvae of this species not only feed but are also able to complete their entire life on the leaves of this Bt poplar (Génissel et al. 2003a; unpublished data). This result contrasts with those obtained for the Colorado potato beetle, Leptinotarsa decemlineata (Say). A colony of this species of the Chrysomelidae was subjected to laboratory selection with Cry3Aa formulations for 29 generations, resulting in a strain 293 times more resistant to this toxin than the wild type (Whalon et al. 1993, Rahardja and Whalon 1995). However, larvae from this strain were unable to feed actively on foliage from transgenic Bt potatoes producing Cry3Aa: they all died within $4 \mathrm{~h}$ (Altre et al. 1996).

Actually, only a fraction of the Bt-resistant insect strains that have artificially been selected are able to feed on the corresponding Bt crop (Tabashnik et al. 2003). The few known examples for which this is the case are all lepidopteran pests: the diamondback moth, Plutella xylostella (L.), the bollworm Heliothis armigera (Hübner), and the pink bollworm, Pectinophora gossypiella (Saunders). Larvae from the CrylCSel strain of P. xylostella, which has a resistance ratio of 12,400 to CrylC, were able to feed on Bt broccoli producing high levels of this toxin (Zhao et al. 2000). Two strain of $H$. armigera, which were resistant to CrylAc, survived on Bt cotton producing this toxin (Fan et al. 2000, Akhurst et al. 2003). Finally, several strains of $P$. gossypiella selected for resistance to CrylAc were also found to be resistant to Bt cotton producing this toxin (Liu et al. 2001, Morin et al. 2003). However, these resistant lepidopteran strains on $\mathrm{Bt}$ crops showed only partial survival, with all strains displaying between 9 and $75 \%$ mortality. Conversely, survival rates were found to be similar in $\mathrm{R} \# 60$ larvae fed Bt poplar foliage and in R\#60 larvae fed nontransgenic hybrid poplar foliage.

The Cry3Aa resistance selected in the $\mathrm{R} \# 60$ strain was almost completely recessive $\left(D_{L C}=0.07\right)$. Reviews by Bourguet et al. (2000) and Ferré and Van Rie (2002) have indicated that for the various Bt resistance genes for which inheritance has been studied,
$D_{L C}$ varies between 0 and 0.88 . The Cry3Aa resistance characterized in this study therefore displays one of the lowest dominance levels ever found for resistance to Bt toxins.

Resistance on transgenic Bt poplar was found to be completely recessive $\left(D_{M L}=0\right)$ because the Bt poplar used in this study produced a concentration of Cry3Aa that was 6.34 times higher than the $\mathrm{LC}_{99}$ of the $\mathrm{F} 1$ hybrids. Resistance selected in the CrylC-Sel and Loxa A strains of P. xylostella was associated with a $D_{L C}$ of 0.39 and 0.16 , respectively (calculated by Ferré and Van Rie 2002). However, resistance to Bt broccoli producing CrylCa (for CrylC-Sel) or CrylAc (for Loxa A) was completely recessive $\left(D_{M L}=0\right)$. Similarly, the resistance to $\mathrm{Bt}$ cotton selected in the APHIS-98R strain of $P$. gossypiella had a $D_{M L}$ of 0 . Thus, all resistance genes discovered to date conferring sufficiently high resistance to allow feeding on $\mathrm{Bt}$ plants (including the resistance to Cry3Aa described here) have been found to have a $D_{M L}$ of 0 . This finding is consistent with the assumption underlying the HDR strategy that heterozygous individuals cannot survive on Bt plants. This is good news because another of the assumptions of the HDR strategy-that alleles conferring resistance to Bt plants are rare in natural pest populations-may not necessarily prove to be true (Gould et al. 1995, Tabashnik et al. 2000, Génissel et al. 2003a).

Finally, our results show that the resistance to the Cry3Aa Bt toxin selected in the $R \# 60$ strain is conferred by a single autosomal gene, with some evidence that the relative proportion of RR individuals within the progeny can be lowered when backcrosses are performed between RS males and RR females. The recessivity of resistance to Cry3Aa toxins is consistent with resistance conferred by an altered target site (Bourguet and Raymond 1998). Because several cadherin alleles have been shown to be associated with $\mathrm{Bt}$ resistance in Heliothis virescens (F.) (Gahan et al. 2001) and P. gossypiella (Morin et al. 2003), cadherin genes are clear candidates for sequence analysis in the near future.

\section{Acknowledgments}

We thank Valerie Dubois for assistance with bioassays, Vincent Sanchis for providing the B. thuringiensis strain, Michel Gohar for help in purifying the Cry3Aa toxin, and Gilles Pilate and Nadège Millet for the multiplication of $\mathrm{Bt}$ poplars. This work was supported by the Appel d'Offre of the Ministère de la Recherche "Impact des Organismes Génétiquement Modifés."

\section{References Cited}

Akhurst, R. J., James, L. Bird, and C. Beard. 2003. Resistance to the Cry1Ac $\delta$-endotoxin of Bacillus thuringiensis in the cotton bollworm, Helicoverpa armigera (Lepidoptera: Noctuidae). J. Econ. Entomol. 96: 1290-1299.

Alstad, D. N., and D. A. Andow. 1995. Managing the evolution of insect resistance to transgenic plants. Science (Wash DC) 268: 1894-1896. 
Altre, J. A., E. J. Grafius, and M. E. Whalon. 1996. Feeding behavior of CryIIIA-resistant and susceptible Colorado potato beetle (Coleoptera: Chrysomelidae) larvae on $\mathrm{Ba}$ cillus thuringiensis tenebrionis-transgenic CryIIIA-treated and untreated potato foliage. J. Econ. Entomol. 89: 311317.

Andow, D. A., and D. N. Alstad. 1998. F2 screen for rare resistance alleles. J. Econ. Entomol. 91: 572-578.

Bourguet, D., and M. Raymond. 1998. The molecular basis of dominance relationships: the case of some recent adaptive genes. J. Evol. Biol. 11: 103-122.

Bourguet, D., A. Génissel, and M. Raymond. 2000. Insecticide resistance and dominance levels. J. Econ. Entomol. 93: 1588-1595.

Bourguet, D., J. Chaufaux, M. Séguin, C. Buisson, J. L. Hinton, T. J. Stodola, P. Porter, G. Cronholm, L. L. Buschman, and D. A. Andow. 2003. Frequency of alleles conferring resistance to $B t$ maize in French and US corn belt populations of the European corn borer, Ostrinia nubilalis. Theor. Appl. Genet. 106: 1225-1233.

Chaufaux, J., M. Marchal, J. Muller Cohn, N. Gilois, and D. Lereclus. 1993. Clônage et expression d'un gène de $B a$ cillus thuringiensis codant pour une delta-endotoxine active contre les larves de Coléoptères. Comptes rendus de l'Académie d'Agriculture de France 79: 103-110.

EPA [Environmental Protection Agency]. 2001. Biopesticides registration action document: Bacillus thuringiensis plant-incorporated protectants $(10 / 16 / 01)$.

Ferré, J., and J. Van Rie. 2002. Biochemistry and genetics of insect resistance to Bacillus thuringiensis. Annu. Rev. Entomol. 47: 501-533.

Finney, D. J. 1971. Probit analysis. Cambridge University Press, London, United Kingdom.

Fan, X., J.-Z. Zhao, Y. Fan, and X. Shi. 2000. Inhibition of transgenic $B t$ plants to the growth of cotton bollworm. Plant Protect. 26: 3-5.

Gahan, L. J., F. Gould, and D. G. Heckel. 2001. Identification of a gene associated with $B t$ resistance in Heliothis virescens. Science (Wash DC) 293: 857-860.

Génissel, A., S. Augustin, C. Courtin, G. Pilate, P. Lorme, and D. Bourguet. 2003a. Initial frequency of alleles conferring resistance to Bacillus thuringiensis poplar in a field population of Chrysomela tremulae. Proc. R. Soc. Lond. B. 270: 791-797.

Génissel, A., J.-C. Leplé, N. Millet, S. Augustin, L. Jouanin, and G. Pilate. 2003b. High tolerance against Chrysomela tremulae of transgenic poplar plants expressing a synthetic cry3aA gene from Bacillus thuringiensis ssp. tenebrionis. Mol. Breed. 11: 103-110.

Georghiou, G. P., and C. E. Taylor. 1977. Operational influences in the evolution of insecticide resistance. J. Econ. Entomol. 70: 653-658.

Gould, F. 1998. Sustainability of transgenic insecticidal cultivars: integrating pest genetics and ecology. Annu. Rev. Entomol. 43: 701-726.

Gould, F., A., Anderson, A., Reynolds, A., Bumgarner, L., and W. J. Moar. 1995. Selection and genetic analysis of $\mathrm{He}$ - liothis virescens (Lepidoptera: Noctuidae) strain with high levels of resistance to Bacillus thuringiensis toxins. J. Econ. Entomol. 88: 1545-1559.

Huang, F. N., Buschman, L. L., Higgins, and H. R. Li. 2002. Survival of Kansas Dipel-resistant European corn borer (Lepidoptera: Crambidae) on Bt and non-Bt corn hybrids. J. Econ. Entomol. 95: 614-621.

James, C. 2003. Global status of commercialized transgenic crops: 2002. ISAAA Briefs No. 27: Preview. ISAAA, Ithaca NY.

Liu, Y. B., B. E. Tabasnik, T. J. Dennehy, A. L. Patin, M. A. Sims, S. K. Meyer, and Y. Carrière. 2001. Effects of Bt cotton and CrylAc toxin on survival and development of pink bollworm (Lepidoptera: Gelechiidae). J. Econ. Entomol. 94: 1237-1242.

Morin, S., R. W. Biggs, M. S. Sisterson, L. Shriver, C. EllersKirk, D. Higginson, D. Holley, L. J. Gahan, D. G. Heckel, Y. Carrière, et al. 2003. Three cadherin alleles associated with resistance to Bacillus in pink bollworm. Proc. Natl. Acad. Sci. U.S.A. 100: 5004-5009.

Rahardja, U., and M. E. Whalon. 1995. Inheritance of resistance to Bacillus thuringiensis subsp. tenebrionis CryIIIA $\delta$-endotoxin in Colorado potato beetle (Coleoptera: Chrysomelidae). J. Econ. Entomol. 88: 21-26.

Raymond, M., G. Prato, and D. Ratsira. 1993. PROBIT analysis of mortality assays displaying quantal response, version 3.3. Praxème Sarl, 34680 St. Georges d'Orques. France.

Robertson, J. L., and H. K. Priesler. 1992. Pesticide bioassays with arthropods. CRC, Boca Raton, FL

Tabashnik, B. E., A. L. Patin, T. J. Dennehy, Y.-B. Liu, Y. Carrière, M. A. Sims, and L. Antilla. 2000. Frequency of resistance to Bacillus thuringiensis in field populations of pink bollworm. Proc. Natl. Acad. Sci. U.S.A. 97: 1298012984.

Tabashnik, B. E., Y. Carrière, T. J. Dennehy, S. Morin, M. S Sisterson, R. T. Roush, A. M. Shelton, and J. Z. Zhao. 2003. Insect resistance to transgenic Bt crops: lesson from the laboratory and crop. J. Econ. Entomol. 96: 1031-1038.

Whalon, M. E., D. L. Miller, R. M. Hollingworth, E. J. Grafius, and J. R. Miller. 1993. Selection of a Colorado potato beetle (Coleoptera: Chrysomelidae) strain resistant to Bacillus thuringiensis. J. Econ. Entomol. 86: 226 233.

Wolfenbarger, L., and P. Phifer. 2000. Biotechnology and ecology - the ecological risks and benefits of genetically engineered plants. Science (Wash DC) 290: 2088-2093.

Zhao, J. Z., H. L. Collins, J. D. Tang, J. Cao, E. D. Earle, R. T. Roush, S. Herrero, B. Escriche, J. Ferré, and A. M. Shelton. 2000. Development and characterization of diamondback moth resistance to transgenic broccoli expressing high levels of Cry1C. Appl. Environ. Microbiol. 66: $3784-3789$

Received 7 October 2003; accepted 12 February 2004 